\title{
Diagnostic Accuracy of Muscle Biopsy and Electromyography in 123 Patients with Neuromuscular Disorders
}

\author{
VASILIOS C. CONSTANTINIDES, MARIA MARTHA PAPAHATZAKI, GEORGIOS K. PAPADIMAS, \\ NIKOS KARANDREAS, THOMAS ZAMBELIS, PANAGIOTIS KOKOTIS and PANAGIOTA MANDA† \\ First Department of Neurology, National and Kapodistrian University of Athens, \\ School of Medicine, Eginition Hospital, Athens, Greece
}

\begin{abstract}
Background/Aim: Diagnostic accuracy of muscle biopsy and electromyography (EMG) in patients with myopathy varies widely among studies. The goal of this study was to examine the diagnostic accuracy of each method in the diagnosis of patients with suspected myopathy, and determine the level of agreement between the two methods. Patients and Methods: The files of all patients with a presumed myopathy were retrospectively reviewed. All patients with detailed muscle biopsy and EMG data were included. Results: A total of 123 patients were included. Accuracy of biopsy was $80.4 \%$ compared to $70.7 \%$ for EMG. Biopsy was sensitive and specific in all neuromuscular disorders. EMG was accurate in neurogenic disorders. Biopsy and EMG agreement was $70.7 \%$. Conclusion: Muscle biopsy is more accurate than EMG in patients with myopathy. Muscle biopsy-EMG discordance can be attributed to different muscle sampling and to disorders with both neurogenic and myopathic features, such as acquired and mitochondrial myopathies.
\end{abstract}

Electromyography (EMG) and muscle biopsy are important in the investigation of patients with neuromuscular disorders. EMG is minimally invasive, provides information on the nature of the underlying process (neurogenic vs. myopathic), and on the duration and distribution of the disease. However, it relies heavily on the expertise of the examiner and rarely gives a precise etiological diagnosis.

This article is freely accessible online.

†Deceased.

Correspondence to: Vasilios C. Constantinides, Eginition Hospital, 7274 Vas. Sofias Avenue, Athens, 11528, Greece. Tel: +30 2107289286, Fax: +30 2107216474, e-mail: vassilis.kon@hotmail.com

Key Words: Muscle, biopsy, electromyography, neuromuscular disorders.
On the other hand, the role of muscle biopsy on the diagnosis of certain neuromuscular diseases, despite some limitations, remains irreplaceable. Agreement between EMG and muscle biopsy varies widely among studies, due to differences in study design (inclusion criteria, sample size and origin, relative representation of diseases in the sample etc.) (1-6). Although methodologically sound, most of these studies present biopsy data with little or no immunohistochemical data, which is routinely performed nowadays and greatly facilitates the accuracy of biopsies. Furthermore, most studies established a final diagnosis based on clinical data (medical history and neurological examination), in conjunction with the biochemical data (muscle enzymes) and conduction studies, without taking into account EMG or muscle biopsy data.

The goal of this study was to present the diagnostic accuracy of muscle biopsy and EMG in patients with a suspected myopathy. We recorded the level of agreement between EMG and muscle biopsy findings. Furthermore, we attempted to investigate whether the level of agreement was affected by differences in muscle sampling and specific myopathy diagnoses.

\section{Materials and Methods}

Patients. The medical files of all patients examined at the Clinic of Neuromuscular Diseases of Eginitio Hospital (First Department of Neurology, National and Kapodistrian University of Athens, Eginition Hospital, Athens, Greece) from 2005 to 2010 were retrospectively reviewed. Only patients with well documented EMG and muscle biopsy data were included in this study. The clinical, biochemical and electrophysiological data (nerve conduction studies, repetitive nerve stimulation etc.) were recorded. No informed consent was required due to the retrospective design of the study. All the patients were submitted to the diagnostic process routinely performed in such cases.

EMG. All EMGs were performed using a Medtronic Functional Diagnostics EMG apparatus (Keypoint net, Skovlunde, Denmark) and concentric needle electrodes (type DCNTM25; Alpine Biomed, Aps, Skovlunde, Denmark), $25 \mathrm{~mm}$ in length and $0.46 \mathrm{~mm}$ in diameter with a recording surface of $0.07 \mathrm{~mm}^{2}$, were used. Skin temperature 
Table I. Distribution of patients based on muscle biopsy findings and final diagnosis.

Final diagnosis, $\mathrm{n}$

\begin{tabular}{lrrrrr}
\cline { 2 - 6 } Muscle biopsy result & Myopathic & Neurogenic & NMJ & Normal & Total \\
\hline Myopathic & 77 & 0 & 1 & 0 & 78 \\
Neurogenic & 5 & 10 & 1 & 0 & 16 \\
Mixed & 1 & 0 & 0 & 0 & 1 \\
Normal & 6 & 0 & 0 & 22 & 28 \\
Total & 89 & 10 & 2 & 22 & 123 \\
\hline
\end{tabular}

NMJ: Neuromuscular junction disorder.

Table II. Distribution of patients based on electromyography (EMG) findings and final diagnosis.

Final diagnosis, $\mathrm{n}$

\begin{tabular}{lcccrc}
\cline { 2 - 6 } EMG result & Myopathic & Neurogenic & NMJ & Normal & Total \\
\hline Myopathic & 68 & 0 & 1 & 13 & 82 \\
Neurogenic & 8 & 10 & 0 & 0 & 18 \\
Mixed & 8 & 0 & 1 & 1 & 10 \\
Normal & 5 & 0 & 0 & 8 & 13 \\
Total & 89 & 10 & 2 & 22 & 123 \\
\hline
\end{tabular}

NMJ: Neuromuscular junction disorder.

was maintained between 31 and $34^{\circ} \mathrm{C}$. Muscles tested by concentric needle differed among patients and were individualized according to the specific clinical question at hand. Insertional activity and spontaneous activity (positive sharp waves, fibrillation potentials, fasciculation potentials) was assessed. Motor unit action potential (MUAP) morphology, duration and amplitude, as well as polyphasia, were compared to our normative data. Interference pattern was characterized as normal or decreased, and recruitment as normal, decreased or early. The time limits of the MUAPs were manually corrected, after inspection of the averaged potentials and re-averaged, thus potentially introducing an element of subjective interpretation.

Biopsy. Only mildly weak muscles (Medical Research Council grade 4/5) (7) were sampled. This was decided in order to avoid end-stage damage. Muscles that were normal during examination were less likely to yield meaningful information when biopsied and were also avoided. Muscle biopsy methodology is described in detail in a previous study of our clinic (8).

Diagnosis. The initial working diagnosis on clinical grounds was that of a possible myopathic disorder, while the final diagnosis was based on the overall assessment of clinical, neurophysiological and pathological parameters and had been established after the unanimous agreement of the group of physicians consisting of an EMG specialist, an expert myopathologist and two clinical neurologists specialized in neuromuscular disorders.
Table III. Sensitivity, specificity, positive ( $P P V)$ and negative predictive value (NPV) of muscle biopsy and electromyography (EMG) according to final diagnosis.

\begin{tabular}{lcccc}
\hline Diagnosis/method & Sensitivity & Specificity & PPV & NPV \\
\hline Myopathy & & & & \\
$\quad$ Muscle biopsy & $86.5 \%$ & $97.1 \%$ & $98.7 \%$ & $73 \%$ \\
$\quad$ EMG & $76.4 \%$ & $58.8 \%$ & $82.9 \%$ & $48.7 \%$ \\
Neurogenic disorder & & & & \\
$\quad$ Muscle biopsy & $100 \%$ & $94.7 \%$ & $62.5 \%$ & $100 \%$ \\
$\quad$ EMG & $100 \%$ & $92.9 \%$ & $55.6 \%$ & $100 \%$ \\
Normal & & & & \\
$\quad$ Muscle biopsy & $100 \%$ & $94.1 \%$ & $78.6 \%$ & $100 \%$ \\
EMG & $57.1 \%$ & $92.1 \%$ & $61.5 \%$ & $87.3 \%$ \\
\hline
\end{tabular}

Table IV. Etiological diagnosis of myopathies in study patients.

\begin{tabular}{lr}
\hline Myopathy analysis & N \\
\hline Acquired & 13 \\
Congenital myopathy & 7 \\
Congenital muscular dystrophy & 1 \\
Metabolic myopathy & 23 \\
Glycogen storage & 7 \\
Lipid acid & 0 \\
Mitochondrial myopathy & 16 \\
Muscular dystrophy & 27 \\
X-linked dystrophinopathy & 1 \\
LGMD & 14 \\
Distal myopathy & 8 \\
Other & 4 \\
Other myopathies & 4 \\
Myotonic dystrophy & 2 \\
Myopathy with tubular aggregations & 2 \\
Unspecified myopathy & 14 \\
Total & 89 \\
\hline
\end{tabular}

LGMD: Limb-girdle muscle dystrophy.

Final EMG and muscle biopsy diagnoses were categorized as myopathic, neurogenic, mixed (i.e. neurogenic and myopathic) and normal. Likewise, the final clinical diagnosis was categorized as a myopathy, a neurogenic disorder, a neuromuscular junction disorder or as normal (i.e. no neuromuscular disorder). Specificity, sensitivity, positive and negative predictive values were calculated for EMG and muscle biopsy for each final clinical diagnostic category.

\section{Results}

Diagnostic accuracy of EMG and muscle biopsy. A total of 123 patients were included. Of these, 89 had a final diagnosis of a myopathy, 10 of a neurogenic disorder, 22 were normal and two had a neuromuscular junction disorder. Diagnostic accuracy of muscle biopsy was 88.6\% (109 of 
Table V. Electromyography (EMG) and muscle biopsy findings in patients with an etiological diagnosis of myopathy.

\begin{tabular}{|c|c|c|c|c|c|c|c|c|c|}
\hline \multirow[b]{2}{*}{ Etiological diagnosis } & \multirow[b]{2}{*}{$\mathrm{N}$} & \multicolumn{4}{|c|}{ EMG, n (\%) } & \multicolumn{4}{|c|}{ Muscle biopsy, n (\%) } \\
\hline & & Myopathic & Neurogenic & Mixed & Normal & Myopathic & Neurogenic & Mixed & Normal \\
\hline Acquired & 13 & $7(54)$ & $1(7)$ & $3(23)$ & $2(15)$ & $11(85)$ & $1(7)$ & $0(0)$ & $1(7)$ \\
\hline Congenital myopathy & 7 & $6(86)$ & $0(0)$ & $0(0)$ & $1(14)$ & $4(57)$ & $1(14)$ & $0(0)$ & $2(29)$ \\
\hline Congenital muscular dystrophy & 1 & $1(100)$ & $0(0)$ & $0(0)$ & $0(0)$ & $1(100)$ & $0(0)$ & $0(0)$ & $0(0)$ \\
\hline Metabolic & 23 & $16(70)$ & $3(13)$ & $2(9)$ & $2(9)$ & $19(83)$ & $2(9)$ & $0(0)$ & $2(9)$ \\
\hline Glycogen storage & 7 & $6(86)$ & $1(14)$ & $0(0)$ & $0(0)$ & $5(71)$ & $1(14)$ & $0(0)$ & $1(14)$ \\
\hline Mitochondrial & 16 & $10(63)$ & $2(13)$ & $2(13)$ & $2(13)$ & $14(88)$ & $1(6)$ & $0(0)$ & $1(6)$ \\
\hline Muscular dystrophy & 27 & $22(81)$ & $3(11)$ & $2(7)$ & $0(0)$ & $26(96)$ & $0(0)$ & $1(4)$ & $0(0)$ \\
\hline X-linked & 1 & $1(100)$ & $0(0)$ & $0(0)$ & $0(0)$ & $1(100)$ & $0(0)$ & $0(0)$ & $0(0)$ \\
\hline Distal & 8 & $7(88)$ & $0(0)$ & $1(12)$ & $0(0)$ & $8(100)$ & $0(0)$ & $0(0)$ & $0(0)$ \\
\hline LGMD & 14 & $11(79)$ & $3(21)$ & $0(0)$ & $0(0)$ & $14(100)$ & $0(0)$ & $0(0)$ & $0(0)$ \\
\hline Other & 4 & $3(75)$ & $0(0)$ & $1(25)$ & $0(0)$ & $3(75)$ & $1(25)$ & $0(0)$ & $0(0)$ \\
\hline Other myopathies & 4 & $3(75)$ & $1(25)$ & $0(0)$ & $0(0)$ & $4(100)$ & $0(0)$ & $0(0)$ & $0(0)$ \\
\hline Myopathy with tubular aggregates & 2 & $2(100)$ & $0(0)$ & $0(0)$ & $0(0)$ & $2(100)$ & $0(0)$ & $0(0)$ & $0(0)$ \\
\hline Myotonic dystrophy & 2 & $1(50)$ & $1(50)$ & $0(0)$ & $0(0)$ & $2(100)$ & $0(0)$ & $0(0)$ & $0(0)$ \\
\hline Unspecified myopathy & 14 & $12(86)$ & $1(7)$ & $1(7)$ & $0(0)$ & $13(93)$ & $0(0)$ & $0(0)$ & $1(7)$ \\
\hline
\end{tabular}

LGMD: Limb-girdle muscle dystrophy.

123). EMG diagnostic accuracy on the other hand was $70.7 \%$ (87 out of 123) (Tables I and II).

Muscle biopsy was highly specific $(97.1 \%)$ and sensitive $(86.5 \%)$ for detection of myopathy. Furthermore, muscle biopsy had $100 \%$ sensitivity and was highly specific for a neurogenic disorder $(94.7 \%)$ or in patients with no neuromuscular disorder $(94.1 \%)$ (Table III).

In contrast, EMG had a moderate sensitivity (76.4\%) and poor specificity $(58.8 \%)$ for a myopathy diagnosis. Likewise, it had poor sensitivity (57.1\%) with high specificity (92.1\%) for patients with no neuromuscular disorder. EMG, however, was highly indicative in cases of neurogenic disorder (sensitivity $100 \%$, specificity $92.9 \%$ ).

Myopathy classification. An etiological diagnosis was reached in $84.2 \%$ of myopathies (75/89). In 14 patients, the underlying myopathy etiology could not be specified. These patients were designated "unspecified myopathy". Of the 75 patients with an etiological diagnosis of a myopathy, 27 had a muscular dystrophy, 23 a metabolic myopathy, 13 had an acquired (inflammatory or toxic) myopathy and seven a congenital myopathy. Other myopathies (e.g. congenital muscular dystrophy, myotonic dystrophy, myopathy with tubular aggregates) were rare (Table IV).

Diagnostic accuracy of EMG and muscle biopsy according to myopathy subtype. Muscle biopsy had $100 \%$ diagnostic accuracy in patients with congenital muscular dystrophy (one case), X-linked (one case), distal (eight cases) and limbgirdle muscular dystrophy (14 cases), as well as in myopathy
Table VI. Electromyography (EMG)-muscle biopsy discrepancies based on final etiological diagnosis.

\begin{tabular}{|c|c|c|c|}
\hline EMG result & Biopsy result & Final diagnosis & $\mathrm{N}(\%)$ \\
\hline Normal & Myopathic & $\begin{array}{l}1 \text { Inflammatory } \\
2 \text { Mitochondrial }\end{array}$ & $3(8)$ \\
\hline Neurogenic & Myopathic & $\begin{array}{l}3 \text { LGMD } \\
1 \text { Mitochondrial } \\
1 \text { Myopathy }\end{array}$ & $5(14)$ \\
\hline Mixed & Myopathic & $\begin{array}{c}3 \text { Acquired } \\
2 \text { Mitochondrial } \\
1 \text { Muscular dystrophy (distal) } \\
1 \text { Myopathy }\end{array}$ & $7(19)$ \\
\hline Mixed & Neurogenic & 1 Anti-MuSK myasthenia & $1(3)$ \\
\hline Mixed & Normal & 1 Normal & $1(3)$ \\
\hline Myopathic & Normal & $\begin{array}{c}13 \text { Normal } \\
1 \text { Metabolic } \\
1 \text { Congenital myopathy } \\
1 \text { Myopathy }\end{array}$ & $16(44)$ \\
\hline Myopathic & Neurogenic & $\begin{array}{c}1 \text { Mitochondrial } \\
1 \text { Myotonic dystrophy } \\
1 \text { Congenital myopathy }\end{array}$ & $3(8)$ \\
\hline
\end{tabular}

Total: 36

LGMD: Limb-girdle muscular dystrophy; MuSK: muscle-specific kinase.

with tubular aggregates (two cases) and myotonic dystrophy (two cases) (Table V).

Muscle biopsy provided mixed results in glycogen storage myopathies (1/7 neurogenic, 1/7 normal), congenital myopathies (1/7 neurogenic, 2/7 normal), mitochondrial myopathies 
Table VII. Electromyography (EMG)-muscle biopsy discrepancies based on agreement of muscle sampling.

\begin{tabular}{|c|c|c|c|c|}
\hline Finding & EMG & Biopsy & Final diagnosis & $\mathrm{N}$ \\
\hline \multirow{6}{*}{ Discordance in same muscle } & & & & 10 \\
\hline & Myopathic & Normal & 3 Normal & 3 \\
\hline & Mixed & Normal & 1 Normal & 1 \\
\hline & Normal & Myopathy & $\begin{array}{c}1 \text { Mitochondrial } \\
1 \text { Acquired (angiitis) }\end{array}$ & 2 \\
\hline & Neurogenic & Myopathy & $\begin{array}{c}1 \text { Mitochondrial } \\
1 \text { Unspecified myopathy }\end{array}$ & 2 \\
\hline & Myopathic & Neurogenic & $\begin{array}{l}1 \text { Myotonic dystrophy } \\
2 \text { Congenital myopathy }\end{array}$ & 3 \\
\hline Concordance in same muscle & & & & 5 \\
\hline Discordance in different muscle & & & & 13 \\
\hline \multirow[t]{2}{*}{ Unavailable muscle } & & & & 8 \\
\hline & & & & Total: 36 \\
\hline
\end{tabular}

(1/16 neurogenic, $1 / 16$ normal) and acquired (inflammatory or toxic) myopathies (1/13 neurogenic, $1 / 13$ normal).

EMG provided mixed results particularly in acquired myopathies (1/13 neurogenic, 3/13 mixed, 2/13 normal), in mitochondrial myopathies (2/23 neurogenic, 2/23 mixed, $2 / 23$ normal) and limb-girdle muscular dystrophy (LGMD) (3/14 neurogenic).

EMG-muscle biopsy agreement: Description of patients with discordant EMG-muscle biopsy findings. Agreement between muscle biopsy and EMG was 70.7\% (87/123). Thirty-six patients had discordant muscle biopsy and EMG findings. In 16 cases, there was a myopathic EMG result with normal biopsy result (one metabolic myopathy, one congenital myopathy, one unspecified myopathy and 13 healthy individuals). Seven patients had an EMG with mixed findings and a myopathic biopsy (three acquired myopathies, two mitochondrial, one distal muscular dystrophy and one unspecified myopathy). In five cases, there was a neurogenic EMG result with myopathic biopsy (three LGMD, one mitochondrial myopathy and one unspecified myopathy) (Table VI).

Analysis of patients with discordant results based on muscles sampled. Of the 36 patients with muscle biopsy and EMG disagreement, only 15 had data on the same (i.e. contralateral) muscle. Of these, five patients had concordant EMG and muscle biopsy data (Table VII).

Ten patients had discordant findings in the same muscle. Four patients with a final diagnosis of no neuromuscular disorder had either a myopathic $(n=3)$ or a mixed $(n=1)$ EMG result with a normal muscle biopsy. Two patients had a normal EMG result with a myopathic biopsy (one mitochondrial myopathy and one acquired myopathy). Two patients had a neurogenic EMG result with myopathic biopsy (one mitochondrial and one unspecified myopathy). Finally, two patients had a myopathic EMG result with a neurogenic biopsy (one myotonic dystrophy and one congenital myopathy).

Thirteen patients had different muscles tested for muscle biopsy and EMG, whereas there were no muscle sample data of muscle biopsy available in eight patients.

\section{Discussion}

This study aimed at presenting the diagnostic accuracy of EMG and muscle biopsy in patients with a suspected myopathy. In our cohort, muscle biopsy provided excellent overall diagnostic accuracy $(\sim 90 \%)$ compared to EMG $(\sim 70 \%)$.

Muscle biopsy was highly sensitive and specific (>85\%) in instances of both neurogenic and myopathic disorders. EMG, on the other hand, was particularly sensitive and specific in patients with a neurogenic disorder (>90\%). However, it lacked specificity in myopathic disorders and sensitivity in patients with no neuromuscular disorder.

Most studies on the subject argue that both EMG and muscle biopsy can provide a diagnostic accuracy of $>90 \%$ in myopathies (1-3). The low diagnostic yield of EMG in our study compared to the literature might be attributed to differences in study design. Most importantly, relevant studies relied solely on the clinical, biochemical and electroneurography data to reach a final diagnosis. In our study, all available data were incorporated to reach a final diagnosis. Although this approach entails the risk of circular-type error, we consider that it provides a more robust final diagnosis.

Interestingly, in our cohort, agreement between EMG and muscle biopsy was moderate $(70.7 \%)$. This ratio is in agreement with the existing literature (ranging from $69.5 \%$ to $82 \%$ ), but low from a clinical standpoint (1-6). 
The main reason for discordance between the two examinations in our study was the presence of 16 patients with myopathic findings at EMG with normal biopsies. This subgroup accounted for $44.4 \%$ of total EMG-biopsy discordance. Eleven out of these sixteen patients had only mild myopathic findings, introducing a level of examiner interpretation. Other studies have also supported that a myopathic EMG result with a normal biopsy represents the commonest subgroup of EMG-biopsy disagreement [13/32 $(40.6 \%)$ and $4 / 6(66.6 \%)$, respectively] $(4,5)$. Of note, there were many patients with the same mild myopathic EMG findings who had a myopathy at biopsy. These results imply that the interpretation of mild myopathic findings in EMG is difficult, even when it is based on quantitative data (numerical values of morphological characteristics of MUAPS). It is possible that there is a degree of overlap in such data between a patient with a slight myopathy and an otherwise completely healthy individual.

Inflammatory myopathies as well as some muscular dystrophies can often present with spontaneous activity in EMG $(9,10)$. Furthermore, mitochondrial myopathies can result in a concomitant neuropathy (particularly of lower limbs) and myopathy (11-13). For this reason, some remaining discrepancies in our cohort are artificial, since they illustrate the dual nature of the aforementioned disorders. This is the case for five patients with mixed EMG and myopathic biopsy findings (three inflammatory and two mitochondrial myopathies); one patient with myopathic EMG and neurogenic biopsy findings (mitochondrial myopathy); one patient with a mitochondrial myopathy with a neurogenic EMG and myopathic biopsy.

Sampling mismatch is the commonest reason for EMGmuscle biopsy discordance, as illustrated in the literature (1$6)$. This is particularly true in diseases with a specific distribution (e.g. proximal more than distal in myopathies, length dependent in axonal neuropathies etc.). Thirteen out of the 36 discordant cases (36\%) in our cohort had different muscles sampled for EMG and biopsy.

Only 10 out of the 36 patients with discordant EMG and biopsy in our cohort had data on the same (i.e. contralateral) muscle. Of these, three involved patients with a normal biopsy and mild myopathic findings in EMG. Two of the remaining patients had a mitochondrial myopathy, one had an inflammatory myopathy, two a congenital myopathy, one a myotonic dystrophy and one an unspecified myopathy. These types of discrepancy might be attributed to the patchy nature of some neuromuscular disorders, such as inflammatory myopathies $(9,10)$. It is possible that the biopsy sample (especially when small in size) was not representative of the underlying process. This is an inherent disadvantage of muscle biopsy that can only be addressed by correct sampling site selection and adequate sample size.
The present study aimed to highlight the diagnostic yield and the possible discrepancies of two classical and wellestablished diagnostic tools for muscle diseases. Since it was of retrospective nature, an accurate diagnosis could not have been made with current sophisticated techniques, such as muscle imaging and next-generation sequencing technology, which have revolutionized the diagnostic approach to muscle diseases.

The distribution of diagnoses differed from what was expected based on epidemiological studies because muscle biopsy and even EMG are not routinely performed in all types of myopathy. For example, in the case of Steinert's disease and facioscapulohumeral muscular dystrophy, the most common inherited muscle disease in adulthood, the diagnosis is usually straightforward by genetic testing.

Our results strengthen the importance of both EMG and muscle biopsy in the work-up of patients with possible neuromuscular disorders. The diagnostic utility of these tests could be further examined by a prospective study incorporating clinical, electrophysiological, biopsy and genetic data. At present, it seems logical to start off with an EMG, which is practically non-invasive, and continue with a muscle biopsy where deemed necessary, particularly when a myopathic disorder is suspected.

\section{References}

1 Hausmanowa-Petrusewicz I and Jedrzejowska H: Correlation between electromyographic findings and muscle biopsy in cases of neuromuscular diseease. J Neurol Sci 13: 85-106, 1971.

2 Buchthal $\mathrm{F}$ and Kamieniecka Z: The diagnostic yield of quantified electromyography and quantified muscle biopsy in neuromuscular disorders. Muscle Nerve 5: 265-280, 1982.

3 Werneck LC and Lima JG: Muscle biopsy correlated with electromyography. Study of 100 cases. Arq Neuropsiquiatr 46: 156-165, 1988.

4 Chang J, Park YG, Choi YC, Choi JH and Moon JH: Correlation of electromyogram and muscle biopsy in myopathy of young age. Arch Phys Med Rehabil 92: 780-784, 2011.

5 Black JT, Bhatt GP, Dejesus PV, Schotland DL and Rowland LP: Diagnostic accuracy of clinical data, quantitative electromyography and histochemistry in neuromuscular disease. A study of 105 cases. J Neurol Sci 21: 59-70, 1974.

6 Rabie M, Jossiphov J and Nevo Y: Electromyography (EMG) accuracy compared to muscle biopsy in childhood. J Child Neurol 22: 803-808, 2007.

7 Medical Research Council: Aids to the Investigation of Peripheral Nerve Injuries. Second Edition. London: Her Majesty's Stationery Office, 1943.

8 Kokotis P, Papadimas GK, Zouvelou V, Zambelis T, Manta P and Karandreas N: Electrodiagnosis and muscle biopsy in asymptomatic hyperckemia. Int J Neurosci 126: 514-519, 2016.

9 Plotz PH, Dalakas M, Leff RL, Love LA, Miller FW and Cronin ME: Current concepts in the idiopathic inflammatory myopathies: Polymyositis, dermatomyositis, and related disorders. Ann Intern Med 111: 143-157, 1989. 
10 Bunch TW: Polymyositis: a case history approach to the differential diagnosis and treatment. Mayo Clin Proc 65: 14801497, 1990.

11 Yiannikas C, McLeod JG, Pollard JD and Baverstock J: Peripheral neuropathy associated with mitochondrial myopathy. Ann Neurol 20: 249-257, 1986.

12 Mizusawa H, Watanabe M, Kanazawa I, Nakanishi T, Kobayashi M, Tanaka M, Suzuki H, Nishikimi M and Ozawa T: Familial mitochondrial myopathy associated with peripheral neuropathy: partial deficiencies of complex I and complex IV. J Neurol Sci 86: 171-184, 1988.
13 Girlanda P, Toscano A, Nicolosi C, Sinicropi S, Picciolo G, Macaione V, Quatrarone A and Messina C: Electrophysiological study of neuromuscular system involvement in mitochondrial cytopathy. Clin Neurophysiol 110: 1284-1289, 1999.

Received June 25, 2018

Revised July 12, 2018

Accepted July 13, 2018 